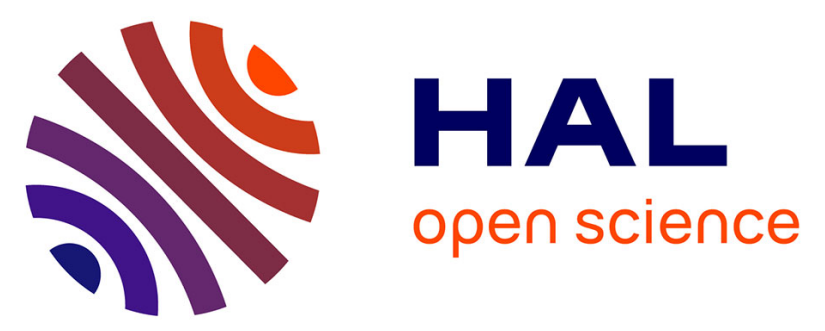

\title{
Self-frequency-doubling Yb:CNGS lasers operating in the femtosecond regime
}

Maciej Kowalczyk, Xuzhao Zhang, Valentin Petrov, Pavel Loiko, Xavier Mateos, Shiyi Guo, Zhengping Wang, Xinguang Xu, Jaroslaw Sotor

\section{- To cite this version:}

Maciej Kowalczyk, Xuzhao Zhang, Valentin Petrov, Pavel Loiko, Xavier Mateos, et al.. Self-frequencydoubling Yb:CNGS lasers operating in the femtosecond regime. Journal of the Optical Society of America B, 2020, 37 (10), pp.2822. 10.1364/JOSAB.399210 . hal-03346030

\section{HAL Id: hal-03346030 \\ https://hal.science/hal-03346030}

Submitted on 12 Oct 2021

HAL is a multi-disciplinary open access archive for the deposit and dissemination of scientific research documents, whether they are published or not. The documents may come from teaching and research institutions in France or abroad, or from public or private research centers.
L'archive ouverte pluridisciplinaire HAL, est destinée au dépôt et à la diffusion de documents scientifiques de niveau recherche, publiés ou non, émanant des établissements d'enseignement et de recherche français ou étrangers, des laboratoires publics ou privés. 


\title{
Self-frequency-doubling Yb:CNGS lasers operating in the femtosecond regime
}

\author{
Maciej KowalczyK, ${ }^{1,},{ }^{*}$ Xuzhao Zhang,${ }^{2,3}$ Valentin Petrov,${ }^{4}$ Pavel \\ LoIKo, ${ }^{5}$ XaVier Mateos, ${ }^{6}$ ShiYI Guo, ${ }^{2}$ Zhengping WANG, ${ }^{2}$ XINGUANG \\ $\mathrm{XU}_{,}{ }^{2}$ AND JAROSŁAW SOTOR ${ }^{1}$ \\ ${ }^{1}$ Laser \& Fiber Electronics Group, Faculty of Electronics, Wroclaw University of Science and \\ Technology, Wybrzeże Wyspiańskiego 27, 50-370 Wrocław, Poland \\ ${ }^{2}$ State Key Laboratory of Crystal Materials, Shandong University, 250100 Jinan, China \\ ${ }^{3}$ Center of Nanoelectronics, School of Microelectronics, Shandong University, Jinan 250100, China \\ ${ }^{4}$ Max Born Institute for Nonlinear Optics and Ultrafast Spectroscopy, Max-Born-Str. 2a, 12489 Berlin, \\ Germany \\ ${ }^{5}$ Centre de Recherche sur les Ions, les Matériaux et la Photonique (CIMAP), UMR 6252 CEA-CNRS- \\ ENSICAEN, Université de Caen Normandie, 6 Boulevard du Maréchal Juin, 14050 Caen Cedex 4, \\ France \\ ${ }^{6}$ Universitat Rovira i Virgili (URV), Física i Cristal-lografia de Materials i Nanomaterials (FiCMA- \\ FiCNA)-EMaS, Marcel.li Domingo 1, 43007 Tarragona, Spain \\ *m.kowalczyk@pwr.edu.pl
}

\begin{abstract}
We study $\mathrm{Yb}: \mathrm{Ca}_{3} \mathrm{NbGa}_{3} \mathrm{Si}_{2} \mathrm{O}_{14}$ (Yb:CNGS) lasers in the mode-locked regime focusing on their self-frequency-doubling potential. We characterize the laser performance in configurations without and with phase-matching for second-harmonic generation. In the former case, the laser generates 47 -fs pulses at a central wavelength of $1052 \mathrm{~nm}$ with an average output power of $45 \mathrm{~mW}$ at $78.4 \mathrm{MHz}$. These pulses are further compressed extracavity down to $40 \mathrm{fs}$, which indicates that the employed crystal is one of the most promising novel gain media regarding ultrafast laser operation. Furthermore, we demonstrate that in a configuration close to phase-matching the laser emits $40 \mathrm{~mW}$ of fundamental radiation at $1046 \mathrm{~nm}$ with 100 -fs pulse duration simultaneously producing $66 \mathrm{~mW}$ of second-harmonic centered at $525 \mathrm{~nm}$. In order to better describe the self-frequency-doubling phenomenon we compare our experimental observations obtained in both configurations with results of numerical modelling.
\end{abstract}

(C) 2020 Optical Society of America under the terms of the OSA Open Access Publishing Agreement

\section{Introduction}

Lasers emitting radiation in the green spectral band have several important emerging applications such as displays [1] or ranging [2]. Moreover, if the radiation is emitted in the form of a high repetition rate train of ultrashort pulses the source can be employed for time-resolved or frequency comb spectroscopy [3]. In case the average power is sufficiently high (> 10-100 $\mathrm{mW}$ ) such green laser sources can be applied for frequency conversion to wavelength ranges otherwise not accessible by ultrashort pulse lasers: e.g. by second-harmonic generation (SHG) to the UV or by synchronous pumping of optical parametric oscillators to the red part of the spectrum [4]. High repetition rate trains of picosecond or femtosecond pulses are normally generated by passive mode-locking of a continuous-wave (CW) laser where the pulse itself modulates the cavity losses, much faster than an external synchronized modulator. However, implementation of this technique to the $\mathrm{Ar}^{+}$ion laser produced only sub-100-ps pulse durations, not much shorter than active mode-locking, basically limited by the narrow spectral lines in gas lasers [5]. Solid-state active media lasing in the green exist, e.g. due to the emission of $\mathrm{Pr}^{3+}$ and $\mathrm{Tb}^{3+}$ ions or upconversion transitions in $\mathrm{Er}^{3+}$-doped crystals, but the corresponding emission bands are also narrow and such passively mode-locked lasers again produced only sub-100-ps pulses [6]. 
Despite the active development of diode-pumped all-solid-state laser sources operating in the visible, the most frequently used scheme for the generation of green light is still based on frequency-doubling of $1-\mu \mathrm{m}$ laser radiation in the process of SHG, because such radiation is readily available. This can be realized in crystals exhibiting non-centrosymmetric structure, which provide the necessary $\chi^{(2)}$ nonlinearity. Interestingly, some of the non-centrosymmetric crystals which possess sufficient birefringence for angle phase-matching can be also used as host matrices for active ions emitting a fundamental wave around $1 \mu \mathrm{m}$, such as neodymium $\left(\mathrm{Nd}^{3+}\right)$ or ytterbium $\left(\mathrm{Yb}^{3+}\right)$. This enables one to design a laser, which emits light in the infrared and simultaneously supports SHG in the visible. Intracavity SHG utilizes the higher fundamental intensity and if the laser is mode-locked, the conversion efficiency will be further enhanced due to the increased peak intensity.

This phenomenon was experimentally observed for the first time in 1969 with a $\mathrm{Tm}^{3+}$-doped $\mathrm{LiNbO}_{3}$ crystal operating at $1853 \mathrm{~nm}$ [7] and was later termed self-frequency-doubling (SFD). Most adequate SFD host materials are obviously non-centrosymmetric crystals containing passive rare-earth ions, such as $\mathrm{Sc}, \mathrm{Y}, \mathrm{La}, \mathrm{Gd}$ or $\mathrm{Lu}$, which can be easily substituted by dopants with the same charge and similar ionic radii. Several such SFD 1- $\mu \mathrm{m}$ Nd lasers have been demonstrated and very efficient $\mathrm{CW}$ operation was obtained in a $\mathrm{Nd}: \mathrm{GdCa}_{4} \mathrm{O}\left(\mathrm{BO}_{3}\right)_{3}$ (Nd:GdCOB) laser emitting green light with an output power up to $3 \mathrm{~W}[8]$. Very recently a slab oscillator based on the same gain medium delivered a record-high power of $17.9 \mathrm{~W}$ at $545.5 \mathrm{~nm}$ [9]. Nevertheless, Nd-based SFD lasers exhibit certain limitations, such as broadband reabsorption of the active ions centered near $530 \mathrm{~nm}$, strongly deteriorating the SHG performance of such sources around their nominal emission wavelength of $1064 \mathrm{~nm}$ [10]. Moreover, neodymium gain media are typically characterized by narrow emission bandwidth, which limits the pulse duration to typically a few picoseconds and hence the peak power that can be achieved in a mode-locked operation. Yet, ultrashort pulse regime is particularly interesting regarding SFD lasers due to the intensity-dependent nature of the SHG process and its interaction with passive mode-locking mechanisms. Moreover, SFD can be employed not only to obtain pulsed visible radiation, but also to introduce negative self-phase modulation based on cascaded $\chi^{(2)}$ nonlinearities. This can be exploited for pulse compression $[11,12]$ or Kerr-lens-like mode-locking [13-15], while such lasers could also become useful tools in applications requiring femtosecond green pulses.

In contrast to $\mathrm{Nd}$ lasers, gain media relying on the $\mathrm{Yb}^{3+}$ ion are basically free from the above-mentioned limitations. This is due to the absence of reabsorption in the visible band (two-level system) and the intrinsically broader gain bandwidth (originating from strong vibronic coupling) enabling generation of sub-100-fs pulses. Even though the broadband emission of $\mathrm{Yb}$-doped materials represents an essential advantage for tunable SFD, it can also become an obstacle on the way towards efficient frequency-doubling. SHG constitutes losses for the fundamental radiation which are intensity-dependent and in the case of mode-locking will counteract this process in any SFD laser. However, if the spectral acceptance of the SHG process is narrower than the broadband gain of the Yb-doped medium, the emission wavelength might escape from it in order to avoid these losses, which will prevent achieving optimal phasematching. This effect is normally absent in $1-\mu \mathrm{m}$ SFD Nd lasers where the SHG spectral acceptance can easily cover the entire emission spectrum. The first CW SFD Yb laser was demonstrated in 1999 using an Yb:GdCOB crystal [16]. Until now, most of the subsequent studies relied on borate crystals such as the isostructural (monoclinic, optically biaxial) oxyborate $\mathrm{Yb}: \mathrm{YCa}_{4} \mathrm{O}\left(\mathrm{BO}_{3}\right)_{3}(\mathrm{Yb}: \mathrm{YCOB})[17](6.2 \mathrm{~W}$ at $513 \mathrm{~nm})$ or the trigonal (uniaxial) $\mathrm{YAl}_{3}\left(\mathrm{BO}_{3}\right)_{4}(\mathrm{Yb}: \mathrm{YAB})[18](1.1 \mathrm{~W}$ at $532 \mathrm{~nm})$. Apart from their relatively high nonlinear coefficients, the calcium rare-earth oxyborates such as YCOB and GdCOB also exhibit extremely broad $\mathrm{Yb}$ emission bandwidths, which enabled the generation of 35-fs pulses at $1055 \mathrm{~nm}$ with $\mathrm{Yb}: \mathrm{YCOB}[19]$.

The majority of the experiments on SFD Yb lasers were devoted to CW operation, including some tunability, and in rare cases Q-switched operation. Only sporadic reports on ultrafast 
lasers can be found in the literature. The latter include three reports on lasers operating under phase-mismatched SHG crystal orientation using: Yb:GdCOB [20] (emitting 90-fs pulses centered at $1045 \mathrm{~nm}$ ), Yb:YCOB (39-fs pulses at $1049 \mathrm{~nm}$ ) [21], and $\mathrm{La}_{\mathrm{x}} \mathrm{Gd}_{\mathrm{y}} \mathrm{Yb}_{\mathrm{z}} \mathrm{Sc}_{\mathrm{w}}\left(\mathrm{BO}_{3}\right)_{4}$ $(x+y+z+w=4)$, shortly Yb:LGSB [22] (180-fs pulses at $1070 \mathrm{~nm})$. However, it has to be noted that the second-harmonic (SH) power levels reported in [21] look highly improbable for the chosen principal crystal cut. To the best of our knowledge, phase-matched SFD in a modelocked $\mathrm{Yb}$ laser has been studied only in one publication. In [23] the authors observed that the SFD mechanism prevented an Yb:YAB laser from standard soliton operation and instead induced generation of strongly chirped picosecond pulses with a total (bidirectional) SH power of $270 \mathrm{~mW}$ at $520 \mathrm{~nm}$. Still, soliton mode-locking with a pulse duration of $245 \mathrm{fs}$ could be achieved under slightly phase-mismatched conditions with an average power of $60 \mathrm{~mW}$ emitted in the visible. Consequently, there is a lack of comprehensive investigation of the SFD mechanism in the femtosecond regime.

Recently, a novel SFD gain medium, Yb:CNGS $\left(\mathrm{Ca}_{3} \mathrm{NbGa}_{3} \mathrm{Si}_{2} \mathrm{O}_{14}\right.$; calcium niobium gallium silicate) has been demonstrated [24]. Yb:CNGS is a langasite-type optically uniaxial (positive) crystal, which belongs to the same trigonal class 32 as YAB and LGSB [25,26], although it shows different structure (space group P321). While the segregation coefficient for $\mathrm{Yb}^{3+}$ is unknown, this dopant ion substitutes a different in charge $\mathrm{Ca}^{2+}$ host-forming cation, which is considered to be the origin of the spectrally broadened bands. The exceptionally broadband emission can potentially match the mode-locked performance of the disordered YCOB/GdCOB [19] or $\mathrm{CaYAlO}_{4} / \mathrm{CaGdAlO}_{4}$ (CALYO/CALGO) crystals [27,28], which enabled generation of the shortest $1-\mu \mathrm{m}$ pulses to date. Indeed, in our preliminary experiments we generated sub-60-fs pulses tunable between 1055 and $1074 \mathrm{~nm}$, still not limited in duration by the intrinsic emission properties of Yb:CNGS [29].

In this work, we present a comprehensive study on an Yb:CNGS laser operating in the mode-locked regime with an emphasis on its SFD properties. In the first part of the paper, we describe the performance of the laser operating in a phase-mismatched SHG configuration. Here we focus on minimizing the pulse duration at the fundamental wavelength of $1052 \mathrm{~nm}$, achieving a 47 -fs pulse width, which could be further compressed extracavity to 40 fs. Moreover, on the basis of computer modelling we show that SFD in this regime leads to generation of two green pulses. The second part of the manuscript is devoted to the investigation of the laser behavior for the case when the SFD effect is maximized. We demonstrate that the mode-locked laser is capable of operating in a standard soliton regime emitting 100-fs pulses at $1046 \mathrm{~nm}$ in a configuration close to phase-matching. Under these conditions it generated radiation at $525 \mathrm{~nm}$ with a total average power of $66 \mathrm{~mW}$. In order to better describe the mechanism of the SFD phenomenon we compare the experimental results with the outcomes of relevant numerical simulations.

\section{Experimental setup}

The schematic of the laser setup is shown in Fig. 1. The active element, 3 at.\% Yb:CNGS (the growth charge composition), had a length of $4 \mathrm{~mm}$ with an aperture of $3 \times 3 \mathrm{~mm}^{2}$, and was antireflective (AR) coated both at the fundamental (F) wavelength of $1050 \mathrm{~nm}$ and its $\mathrm{SH}$ of $525 \mathrm{~nm}$. The crystal was cut for type-I (ee-o) phase-matched SHG at $1064 \mathrm{~nm}$ with the angles $\varphi=90^{\circ}$ and $\theta=36^{\circ}$ in the orthogonal $x y z$ frame where $x \equiv a$ and $z \equiv c$ ( $a$ and $c$ are crystallographic axes). Note that type-I phase-matching (for CNGS $d_{\mathrm{eff}}=d_{11} \cos ^{2} \theta \sin 3 \varphi$ ) is a prerequisite for efficient SFD because spatial and temporal walk-off effects are absent between the two input $F$ waves. No cooling of the crystal was provided.

As a pump source we employed a laser diode, which emitted up to $1 \mathrm{~W}$ of $979.4 \mathrm{~nm}$ radiation. The pump beam was delivered via a single-mode polarization-maintaining fiber and reimaged onto the gain medium with a resulting focal radius of $22 \mu \mathrm{m}$ and a confocal parameter of $5.2 \mathrm{~mm}$ inside the crystal. The pump polarization was aligned as horizontal forming an $e$-ray inside the crystal, which was placed at a normal incidence between two 100-mm spherical 
mirrors with its $c$-axis in the horizontal plane. These mirrors were AR-coated at the pump wavelength, highly-reflective (HR)-coated at the Flaser wavelength and provided a transmittance of $65 \%$ at the $\mathrm{SH}$ of the latter.

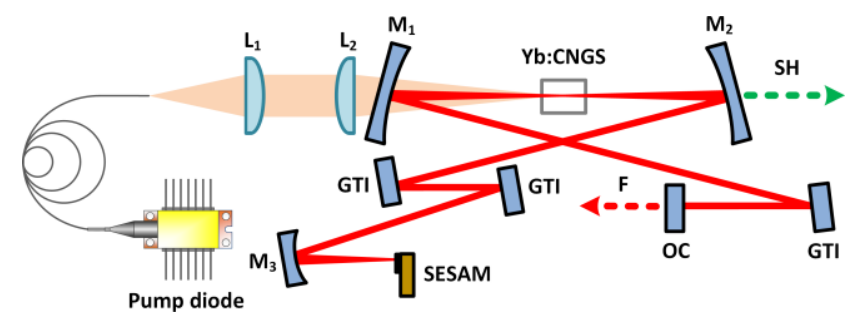

Fig. 1. Schematic layout of the Yb:CNGS laser: $\mathrm{L}_{1}-18.4 \mathrm{~mm}$ aspheric lens; $\mathrm{L}_{2}-100 \mathrm{~mm}$ spherical lens; $\mathrm{M}_{1-3}-100 \mathrm{~mm}$ concave spherical mirror; GTI — Gires-Tournois-Interferometer mirror; OC - output coupler; SESAM - semiconductor saturable absorber mirror; F fundamental wavelength output; $\mathrm{SH}$ - second harmonic output.

For mode-locking we adopted a hybrid regime exploiting two types of intensity-dependent loss mechanisms [30]. In order to initiate the pulsed operation we focused the intracavity beam onto a semiconductor saturable absorber mirror (SESAM) with a resulting beam radius of $30 \mu \mathrm{m}$ (estimated with the ABCD matrix formalism). The finite relaxation time of the SESAM enabled self-starting operation with a pulse duration in the range of $100 \mathrm{fs}$. Subsequently, we carefully decreased the distance between the SESAM and $\mathrm{M}_{3}$ mirror pushing the cavity towards the edge of the stability range. This affected the beam size within the gain medium and activated a Kerr-lensing mechanism, further shortening the duration of the generated pulses due to its quasi-instantaneous response. In order to obtain stable mode-locked operation we had to counterbalance the positive group-delay dispersion of the crystal (single-pass $\mathrm{GDD}=330 / 380 \mathrm{fs}^{2}$ for $o / e\left(\theta=36^{\circ}\right)$-ray, respectively [31]) and the effect of self-phase modulation (SPM) induced therein. Negative dispersion was introduced with three different Gires-Tournois Interferometer (GTI) mirrors with a single-bounce GDD of $-100,-250$, and $-550 \mathrm{fs}^{2}$ (Layertec) used in a double pass. This specific configuration was experimentally optimized regarding the shortest achievable pulse duration. Higher values of negative GDD led to generation of longer pulses, while for insufficient GDD we observed mode-locking in the chirped pulse oscillator regime [32,33]. The total physical length of the X-shaped cavity amounted to $190 \mathrm{~cm}$.

\section{Results}

\subsection{Non-phase-matched configuration}

Initially, we investigated the laser performance without SHG phase-matching. For this experiment we employed an OC with a transmission of $1 \%$ and a SESAM (BATOP) with a finite relaxation time of $1 \mathrm{ps}$ and a modulation depth of $1.2 \%$. The cavity was aligned to enforce lasing with $\sigma$ polarization ( $o$-ray inside the crystal) in order to exploit the highest gain of the Yb:CNGS crystal [26]. This was possible through fine alignment of the overlap with the pump beam due to the spatial walk-off of the two eigen-polarizations inside the crystal (Poynting vectors).

Figure 2 illustrates the performance of the laser operating in the most broadband regime. It was characterized with an optical spectrum analyzer (OSA, AQ6370, Yokogawa) and a SH frequency-resolved optical gating (FROG) device (Mesa Photonics). Emitted spectrum exhibited peak wavelength of $1052 \mathrm{~nm}$ and $28 \mathrm{~nm}$ full-width-at-half-maximum (FWHM) bandwidth. However, it was strongly broadened in the long wavelength wing. The calculated Fourier-limited pulse duration for the measured spectrum amounts to $36 \mathrm{fs}$ (see Fig. 2b). The FROG-retrieved pulse duration of $47 \mathrm{fs}$ indicated residual chirp, which is confirmed by the spectral phase profile depicted in Fig. 2a. While the phase was almost flat within the main pulse 
component, the long-wavelength tail was clearly upchirped (the chirp sign was identified by a subsequent measurement after introducing additional GDD of a known value). This was caused by the imperfect dispersion compensation with GTI mirrors characterized with limited spectral band up to approximately $1065 \mathrm{~nm}$. Subsequently, we managed to partially compress the pulses with two extracavity bounces on $-250 \mathrm{fs}^{2}$ GTI mirrors. This obviously introduced an additional chirp to the main pulse component (see Fig. 2a), but ultimately enabled the reduction of the pulse duration down to $40 \mathrm{fs}$. It has to be emphasized that an intensity autocorrelation measurement of the pulse duration indicated a slightly shorter value of $38 \mathrm{fs}$, mainly due to the pulse shape deviation from a fitted $\mathrm{sech}^{2}$ profile. Additionally, in the inset of Fig. $2 \mathrm{~b}$ we present a radio-frequency $(\mathrm{RF})$ spectrum of the fundamental beat note at $78.4 \mathrm{MHz}$. It exhibits a signalto-noise ratio of $70 \mathrm{~dB}$ confirming the stability of the mode-locked operation. The average output power emitted from the oscillator amounted to $45 \mathrm{~mW}$.
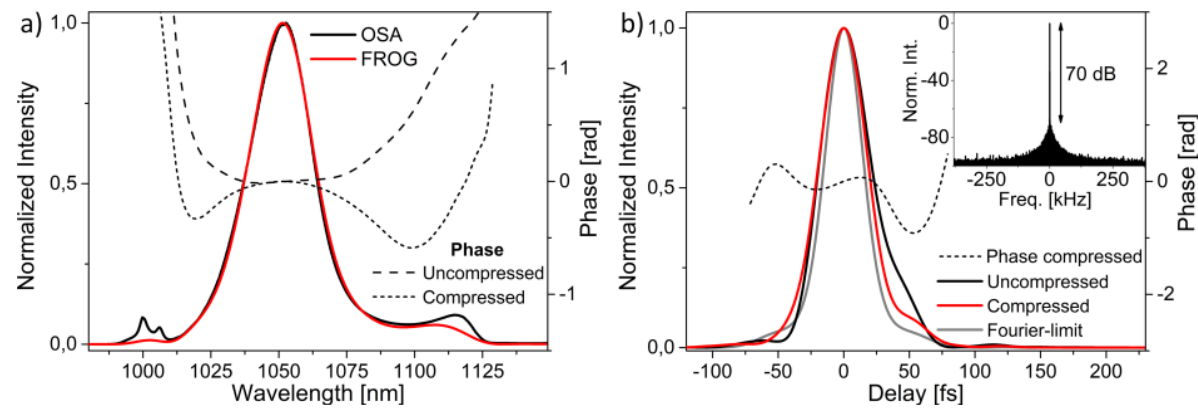

Fig. 2. Performance of the $\mathrm{Yb}$ :CNGS laser in the non-phase-matched SFD configuration: optical spectrum recorded with a standard OSA and retrieved from a measured FROG trace; spectral phase with and without extracavity compression (a); FROG-retrieved pulse profile with and without compression compared with the calculated Fourier-limited pulse; temporal phase of the compressed pulses, inset: RF spectrum of the fundamental beat note at $78.4 \mathrm{MHz}$ (b).

We believe that the achieved reduction of the pulse duration when compared with our previous experiments [29] originated mainly from the different crystal sample employed. The normal incidence operation enabled higher intensity resulting in an enhanced SPM contribution that was additionally increased by the longer crystal length. Moreover, we expect that the pulse duration can be further shortened by applying high brightness pumping with, e.g. a fiber laser [27].

Regardless of the lack of phase-matching conditions for SFD, we observed residual SHG from the fundamental 1050-nm beam. This is due to the type-0 (oo-o) process, involving the single nonlinear coefficient $d_{11}$ of CNGS. None of the folding cavity mirrors was HR-coated for $525 \mathrm{~nm}$, therefore the strongest SH signal was registered directly after one of them, mirror $\mathrm{M}_{2}$. Figure 3a shows the SH spectrum measured with a CCS100 (Thorlabs) spectrometer. The spectrum exhibited characteristic fringes, which originated from the generation of two interfering SH pulses. Double pulse generation is a direct consequence of the absence of both phase- and group-velocity-matching between the F and SH beams in the process of SFD [3436]. This mechanism is illustrated with the results of $1 \mathrm{D}$ numerical modelling performed with the Hussar software [37]. Spatially plane waves were assumed in the model with the spatial walk-off $\left(\sim 1.6^{\circ}\right)$ neglected. While the SFD properties of Yb:CNGS have been investigated previously [24], the value of the nonlinear coefficient $d_{11}$ was not measured. Here, for the purpose of visualization, we used a value determined for a similar isostructural compound, $\mathrm{Ca}_{3} \mathrm{TaGa}_{3} \mathrm{Si}_{2} \mathrm{O}_{14}$ (CTGS), which amounts to $0.72 \mathrm{pm} / \mathrm{V}$ [38]. The reliability of this value is discussed in the next section devoted to phase-matched SFD. However, for the case of strong phase- and group-velocity mismatch the exact CNGS nonlinearity is unimportant and changing the $d_{11}$ value does not introduce any significant modifications to the simulation outcomes. Figures $3 \mathrm{c}$ and $3 \mathrm{~d}$ show the evolution of the temporal distribution of the two SH pulses as they propagate along the $\mathrm{Yb}$ :CNGS crystal. The time delay is normalized to the position of the 
F pulse, indicating that one of the green pulses is synchronized with it, while the second one is delayed. This is also demonstrated in Fig. $3 \mathrm{~b}$ depicting the profile of both F and SH beams at the gain medium exit.
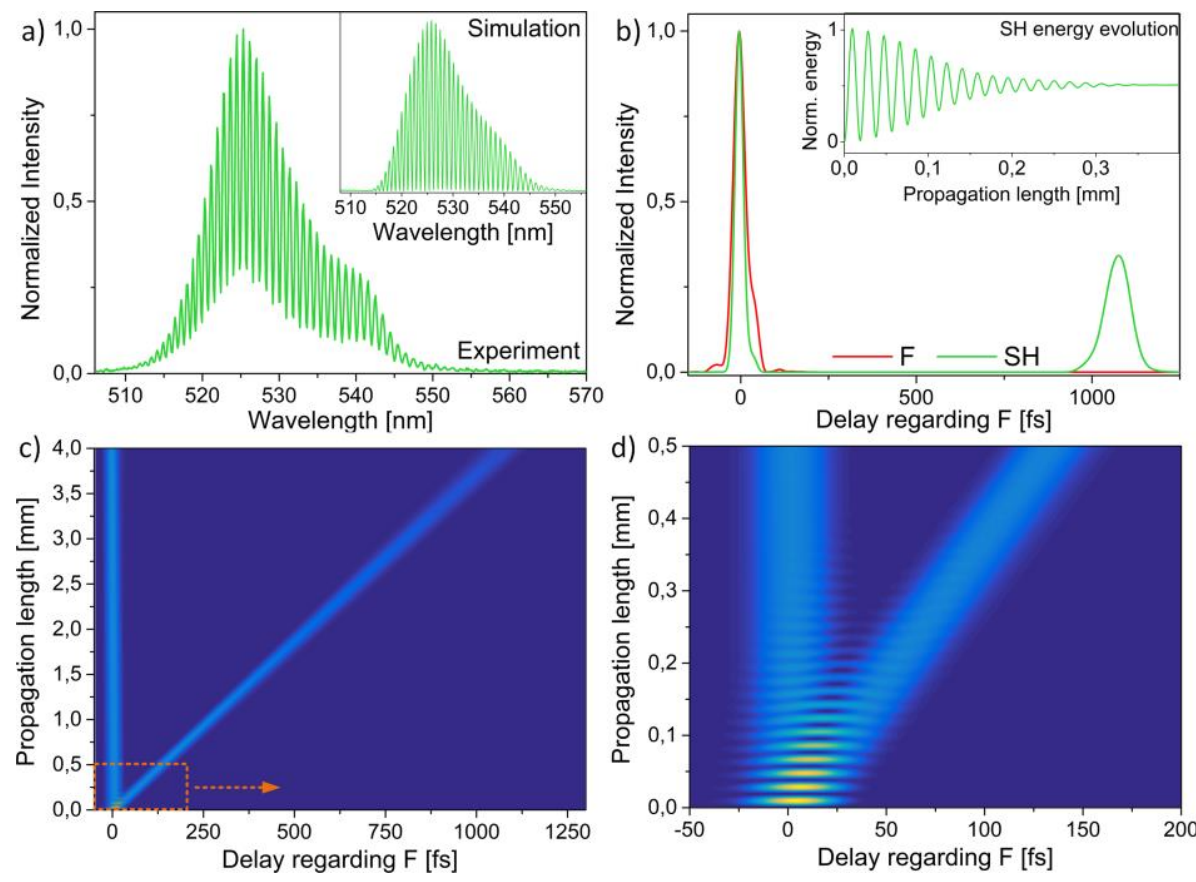

Fig. 3. Measured SH spectrum of the Yb:CNGS laser in the non-phase-matched SFD configuration, inset: simulated SH spectrum (a); simulated temporal profile of the fundamental (F) and second harmonic (SH) pulses at the crystal exit, inset: normalized SH pulse energy evolution (b); simulated evolution of the SH signal temporal profile along the crystal (c); the area marked with a dashed line is magnified on (d).

The origin of this behavior can be briefly summarized as follows. At the entrance of the crystal the SH starts to build up, but after covering the first coherence length $\left(L_{c o h}=9.5 \mu \mathrm{m}\right)$ it converts back to the $\mathrm{F}$ (causing the SH pulse energy oscillations visible in the inset of Fig. 3b). Yet, due to different group velocities of the two beams, a trailing edge of the SH component does not overlap temporally with the $\mathrm{F}$ beam anymore. Thus, it cannot be back-converted and gets isolated with a relative delay to the $\mathrm{F}$ pulse defined by their group-velocity mismatch and the propagation length. Due to the same process, the leading edge of the F pulse constantly generates SH but does not experience any of its back-conversion. Consequently, the SH signal is periodically generated and back-converted as it travels under the envelope of the F pulse. After an initial period, these two mechanisms balance each other and the energy of this SH pulse is stabilized (see inset in Fig. 3b).

Due to the crystal orientation ( $\varphi=0$ for an $o$-ray of the F beam), the SH beam has the same polarization as the driving F radiation (oo-o process). Because the non-phase-matched SHG is obviously not frequency-dependent, it enabled the conversion of the entire spectrum of the sub50 -fs pulses. Simultaneously, it strongly hampered the conversion efficiency, limiting the SH average power to the microwatt level.

\subsection{Phase-matched configuration}

Subsequently, we investigated the laser behavior with maximized SFD effect. For this, the cavity was realigned to enforce lasing of the $\mathrm{F}$ beam with $\pi$ polarization enabling phasematched type-I SHG (ee-o) conditions. While the SFD loss was negligible under phasemismatched conditions, it became crucial for the $1050-\mathrm{nm}$ fundamental radiation as we strongly 
increased the conversion efficiency. The mode-locked regime was stabilized by the introduced SESAM and Kerr-lensing, which favored high-intensity pulsed operation over CW lasing. Because the SFD is an instantaneous intensity-dependent process, it naturally counteracts the action of the passive mode-locking mechanisms and can eventually destabilize mode-locking. In order to balance this effect, we employed a SESAM with the highest available modulation depth of $2.6 \%$ (relaxation time of $500 \mathrm{fs}$; BATOP). We have indeed observed strong improvement of the mode-locking stability under conditions close to phase-matching, when the SESAM modulation was increased. Moreover, an $\mathrm{OC}$ with a transmittance of $0.8 \%$ was used to minimize the intracavity losses.
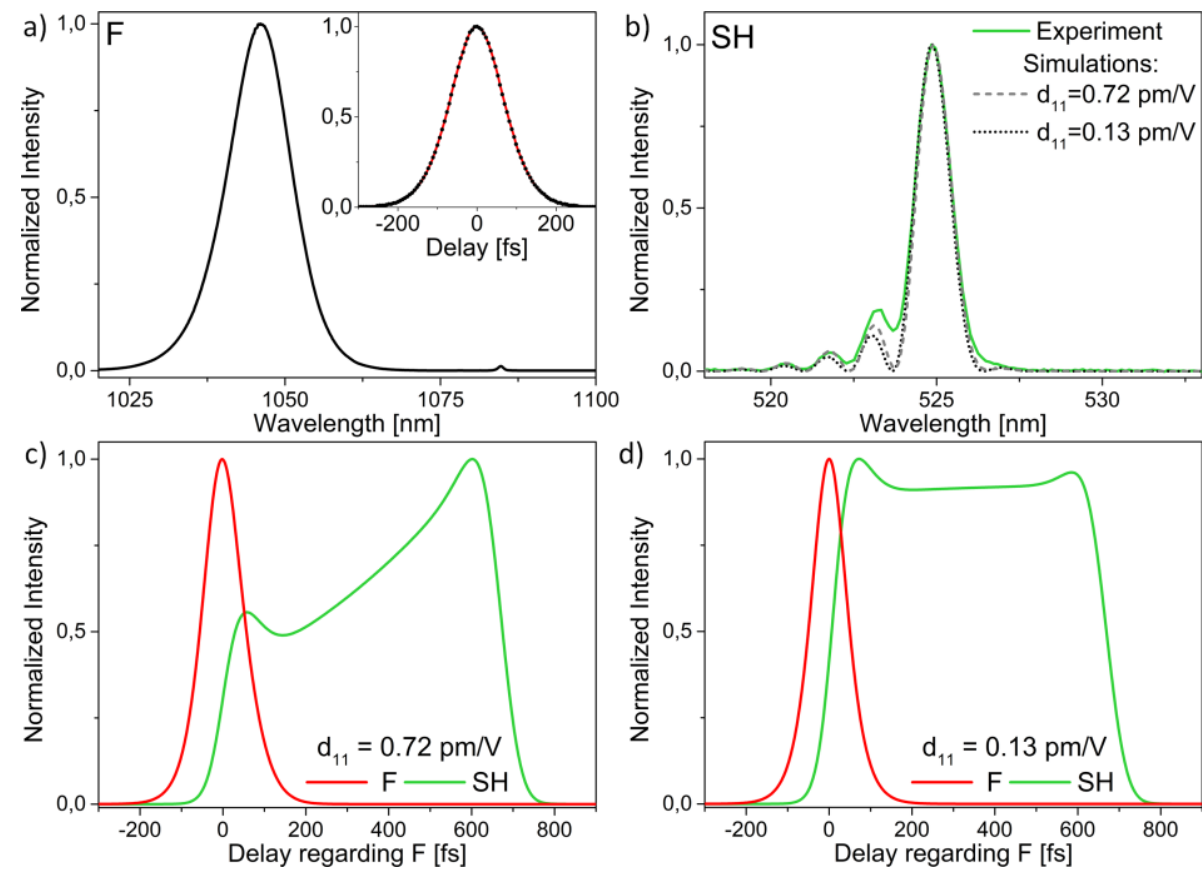

Fig. 4. Performance of the Yb:CNGS laser in the SFD configuration close to phase-matching: optical spectrum, inset: an autocorrelation trace with a fit assuming $\operatorname{sech}^{2}$ pulses (a); measured second harmonic spectrum compared with the results of the numerical modelling assuming two different values of the $d_{11}$ coefficient (b); simulated temporal profile of the $\mathrm{F}$ and SH pulses (independently normalized) at the crystal output assuming $d_{11}=0.72 \mathrm{pm} / \mathrm{V}$ (c) and $d_{11}=0.13 \mathrm{pm} / \mathrm{V}(\mathrm{d})$.

Typically, the mode-locked operation was self-starting when the pump power exceeded the threshold of approximately $500 \mathrm{~mW}$. The corresponding average output power of the SH beam measured directly after $\mathrm{M}_{2}$ was in the range of $1-5 \mathrm{~mW}$. The still low conversion efficiency was associated with the central wavelength of the fundamental laser emission which moved away from the exact phase-matching in order to avoid the SFD loss introduced to the 1- $\mu \mathrm{m}$ $F$ radiation. Subsequently, we changed the effective $\theta_{\text {eff }}$ angle between the propagation vector and the optical axis of $\mathrm{Yb}$ :CNGS by fine-tilting the crystal and/or one of the cavity end mirrors. As $\theta_{\text {eff }}$ was tuned to maximize the SH output power, we observed two different effects: the increased SFD loss destabilized the mode-locked operation or it resulted in a further shift of the central emission wavelength out of the phase-matched configuration. Consequently, we were not able to achieve mode-locking with perfect phase-matching for the peak wavelength of the fundamental emission. The highest power of the SH beam recorded after $\mathrm{M}_{2}$ mirror amounted to $21.4 \mathrm{~mW}$. This indicates that the SH power directly after the crystal was equal to $33 \mathrm{~mW}$ and the total SH power generated in both directions amounted to $66 \mathrm{~mW}$ with $42.8 \mathrm{~mW}$ emitted 
from the cavity. The latter value can be easily increased without affecting the laser performance by employing $\mathrm{SH}$ output mirrors $\left(\mathrm{M}_{1,2}\right)$ with low reflectance in the visible band.

In this configuration the laser was still operating in a stable soliton mode-locking regime emitting 102-fs pulses with $11.8 \mathrm{~nm}$ broad spectrum centered at $1046 \mathrm{~nm}$. Figure 4a shows recorded spectrum of the fundamental radiation and an autocorrelation trace with a fit assuming $\mathrm{sech}^{2}$ pulse profile (obtained with APE PulseCheck). The average output power of the $\mathrm{F}$ beam was equal to $40 \mathrm{~mW}$, indicating that the double pass SHG conversion efficiency amounted to roughly $1.3 \%$. Note that this value exceeds the OC losses, but it is still lower than the modulation depth of the employed SESAM. The laser performance has been characterized for the pump power of $950 \mathrm{~mW}$ and the mode-locked operation could be sustained for a whole range of pump power levels between $\sim 500 \mathrm{~mW}-950 \mathrm{~mW}$.

Figure $4 \mathrm{~b}$ presents the recorded SH spectrum with a central wavelength of $524.9 \mathrm{~nm}$. This means that the crystal was effectively configured for phase-matched SHG at $1049.8 \mathrm{~nm}$, shifted by almost $4 \mathrm{~nm}$ from the center of the $\mathrm{F}$ spectral peak. This is equivalent to a $-0.2^{\circ}$ deviation from the optimal phase-matching angle. We have compared the experimental results with the outcomes of the 1D numerical modeling based on the experimental conditions. In contrast to the phase-mismatched case, which was discussed in the previous section, here the nonlinearity of the CNGS crystal is a crucial parameter. First, as the $d_{11}$ coefficient is unknown for the studied crystal, we again applied the value measured for CTGS, i.e. $d_{11}=0.72 \mathrm{pm} / \mathrm{V}$ [38]. For comparison with the experiment, we used a spatially averaged intensity ( $1 / 2$ of the peak value) in the 1D model. The simulated SH spectrum for such a nonlinear process (dashed grey line in Fig. 4b) is consistent with the experimental data. The measured spectral bandwidth of the main component amounted to $1.2 \mathrm{~nm}$ which agrees with a $1.19-\mathrm{nm}$ acceptance bandwidth calculated for the 4-mm long $\mathrm{Yb}: \mathrm{CNGS}$ crystal at $525 \mathrm{~nm}$. The Fourier-limited pulse duration for the given bandwidth and a sech ${ }^{2}$ pulse profile amounts to $240 \mathrm{fs}$. Nevertheless, a sech ${ }^{2}$ shape cannot be applied for the investigated SFD mechanism, because of the group-velocity-mismatch between the F and SH beams inside the crystal. The SH pulse constantly drains energy from the F pulse, but it also temporally lags behind it as both waves propagate along the crystal. Consequently, the temporal distribution of the generated SH signal strictly depends on its nonlinear interaction with the F component. This can be clearly seen in Fig. 4c, which shows profiles of both beams at the crystal exit. It yields a SH pulse duration (FWHM) of $230 \mathrm{fs}$, but with a long low-intensity tail, which occurs because of a partial depletion of the $\mathrm{F}$ beam. However, the conversion efficiency calculated for the described process amounts to $13.5 \%$, which clearly contradicts the experimentally estimated value of $0.66 \%$ for a single pass. Since our simulations show that the spatial walk-off effect has a minor effect on the conversion efficiency under described experimental conditions, we believe that this discrepancy originated from a highly overestimated value of the nonlinear coefficient. A similar conclusion can be found in a previous study on Nd:CNGS [39], where the authors estimated the upper limit of the $d_{11}$ value to be on the order of $0.46 \mathrm{pm} / \mathrm{V}$. Here, we matched the calculated conversion efficiency to the actually observed level by assuming much lower nonlinear coefficient $\left(d_{11}=0.13 \mathrm{pm} / \mathrm{V}\right)$. The SH pulse shape would be then close to a flat-top $(\mathrm{FWHM}=670 \mathrm{fs})$, due to the negligible depletion of the F beam under these conditions (Fig. 4d). Note that in such a case the pulse duration is defined by the group velocity mismatch and the crystal length. Simultaneously, assuming much lower CNGS nonlinearity does not significantly affect the spectrum (dotted black line in Fig. 4b).

\section{Conclusion}

In conclusion, we have realized a mode-locked Yb:CNGS laser operating in the femtosecond regime and studied its SFD properties in configurations without and with SHG phase-matching. When the laser was operated under the conditions preventing efficient nonlinear frequency conversion, it generated pulses centered at $1052 \mathrm{~nm}$ with a duration of $47 \mathrm{fs}$ and an average power of $45 \mathrm{~mW}$, which were subsequently compressed extracavity to $40 \mathrm{fs}$. Note that this 
achievement still does not exploit the entire gain spectrum of $\mathrm{Yb}$ :CNGS, which indicates that this material is one of the most promising novel crystals concerning ultrashort pulse generation near $1 \mu \mathrm{m}$. Moreover, we characterized the non-phase-matched SH signal and compared the experimental observations with the outcomes of computer modelling, confirming that this regime results in generation of two ultrashort green pulses. In the second part, we reconfigured the laser in order to enable efficient type-I SHG and maximize the power emitted in the visible band. We were able to tune the central wavelength $(1046 \mathrm{~nm})$ of the fundamental radiation down to $4 \mathrm{~nm}$ out of optimal phase-matching. In this case, the laser emitted 100 -fs soliton pulses with an average power of $40 \mathrm{~mW}$ and it simultaneously generated $66 \mathrm{~mW}$ of radiation at $525 \mathrm{~nm}$ (with $\sim 43 \mathrm{~mW}$ emitted from the cavity). On the basis of numerical simulations, we have also studied the relation between the $\mathrm{Yb}$ :CNGS nonlinearity and the temporal shape of the SH pulse.

We were unable to achieve perfect phase-matching for the peak wavelength of the infrared radiation. As in all $\mathrm{Yb}$ lasers, this can be attributed to the broad gain bandwidth of the crystal, which facilitated spectral shift of the fundamental emission wavelength when the crystal was tilted towards phase-matching, to avoid the losses. This effect can be suppressed by locking the spectrum beneath a broader phase-matching acceptance bandwidth. The latter will require reduction of the crystal thickness to about $1 \mathrm{~mm}$ and higher doping level to recover the pump absorption in Yb:CNGS. Another important conclusion from the present study is that low second order nonlinearity (as observed in CNGS) is not a serious limitation in SFD lasers if they operate in the femtosecond regime. Thus sufficiently high conversion efficiency into the visible can be expected with a shorter crystal operating in the gain maximum. The main advantage of $\mathrm{Yb}$ :CNGS over similar SFD crystals is its capability to produce very short pulses at the fundamental wavelength, which is expected to result in sub-100-fs durations with sufficient average power in the green in the near future.

\section{Funding}

National Science Centre (NCN, Poland) (2015/18/E/ST7/00296); National Natural Science Foundation of China (61975096).

\section{Acknowledgments}

We would like to acknowledge Laser Zentrum Hannover for fabrication of the AR coatings on the $\mathrm{Yb}$ :CNGS sample. Moreover, we would like to thank Yicheng Wang (Ruhr University Bochum) for the insightful discussions and Tomasz Kardaś (Fluence) for the help with his software Hussar, which is freely available at www.ufs.edu.pl. The purchase of the FROG equipment was supported by the Foundation for Polish Science within the First TEAM program co-financed by the European Union under the European Regional Development Fund (contract no. POIR.04.04.00-00-434D/17-00).

\section{Disclosures}

The authors declare no conflicts of interest.

\section{References}

1. K. V. Chellappan, E. Erden, and H. Urey, "Laser-based displays: A review," Appl. Opt. 49, F79-F98 (2010).

2. J. F. McGarry, E. D. Hoffman, J. J. Degnan, J. W. Cheek, C. B. Clarke, I. F. Diegel, H. L. Donovan, J. E. Horvath, M. Marzouk, A. R. Nelson, D. S. Patterson, R. L. Ricklefs, M. D. Shappirio, S. L. Wetzel, and T. W. Zagwodzki, "NASA's satellite laser ranging systems for the twenty-first century," J. Geod. 93, 2249-2262 (2019).

3. T. Ideguchi, A. Poisson, G. Guelachvili, T. W. Hänsch, and N. Picqué, "Adaptive dual-comb spectroscopy in the green region," Opt. Lett. 37, 4847-4849 (2012).

4. V. Petrov, M. Ghotbi, O. Kokabee, A. Esteban-Martin, F. Noack, A. Gaydardzhiev, I. Nikolov, P. Tzankov, I. Buchvarov, K. Miyata, A. Majchrowski, I. V. Kityk, F. Rotermund, E. Michalski, and M. Ebrahim-Zadeh, "Femtosecond nonlinear frequency conversion based on $\mathrm{BiB}_{3} \mathrm{O}_{6}$," Laser Photonics Rev. 4, 53-98 (2010).

5. Y. Wang and E. Bourkoff, "Passive mode locking of the Ar ${ }^{+}$laser," Appl. Opt. 27, 2655-2661 (1988).

6. Y. Zhang, H. Yu, H. Zhang, A. Di Lieto, M. Tonelli, and J. Wang, "Laser-diode pumped self-mode-locked praseodymium visible lasers with multi-gigahertz repetition rate," Opt. Lett. 41, 2692-2695 (2016). 
7. L. F. Johnson and A. A. Ballman, "Coherent emission from rare earth ions in electro-optic crystals," J. Appl. Phys. 40, 297-302 (1969).

8. H. Yu, N. Zong, Z. Pan, H. Zhang, J. Wang, Z. Wang, and Z. Xu, "Efficient high-power self-frequencydoubling Nd:GdCOB laser at 545 and $530 \mathrm{~nm}$," Opt. Lett. 36, 3852-3854 (2011).

9. J. Du, J. Wang, H. Yu, and H. Zhang, "17.9 W continuous-wave self-frequency-doubled Nd:GdCOB laser," Opt. Lett. 45, 327-330 (2020).

10. H. Yu, Z. Pan, H. Zhang, and J. Wang, "Recent advances in self-frequency-doubling crystals," J. Materiomics 2, 55-65 (2016).

11. G. I. Stegeman, D. J. Hagan, and L. Torner, " $\chi^{(2)}$ cascading phenomena and their applications to all-optical signal processing, mode-locking, pulse compression and solitons," Opt. Quantum Electron. 28, 1691-1740 (1996).

12. C. R. Phillips, A. S. Mayer, A. Klenner, and U. Keller, "SESAM modelocked Yb:CaGdAlO ${ }_{4}$ laser in the soliton modelocking regime with positive intracavity dispersion," Opt. Express 22, 6060-6077 (2014).

13. M. Zavelani-Rossi, G. Cerullo, and V. Magni, "Mode locking by cascading of second-order nonlinearities," IEEE J. Quantum Electron. 34, 61-70 (1998).

14. S. J. Holmgren, V. Pasiskevicius, and F. Laurell, "Generation of $2.8 \mathrm{ps}$ pulses by mode-locking a Nd:GdVO ${ }_{4}$ laser with defocusing cascaded Kerr lensing in periodically poled KTP," Opt. Express 13, 5270-5278 (2005).

15. A. S. Mayer, C. R. Phillips, and U. Keller, "Watt-level 10-gigahertz solid-state laser enabled by self-defocusing nonlinearities in an aperiodically poled crystal," Nat. Commun. 8, 1673/1-8 (2017).

16. F. Mougel, K. Dardenne, G. Aka, A. Kahn-Harari, and D. Vivien, "Ytterbium-doped $\mathrm{Ca}_{4} \mathrm{GdO}\left(\mathrm{BO}_{3}\right)_{3}$ : an efficient infrared laser and self-frequency doubling crystal," J. Opt. Soc. Am. B 16, 164-172 (1999).

17. D. Lu, Q. Fang, X. Yu, X. Han, J. Wang, H. Yu, and H. Zhang, "Power scaling of the self-frequency-doubled quasi-two-level Yb:YCOB laser with a 30\% slope efficiency," Opt. Lett. 44, 5157-5160 (2019).

18. P. Dekker, J. M. Dawes, J. A. Piper, Y. Liu, and J. Wang, "1.1 W CW self-frequency-doubled diode-pumped $\mathrm{Yb}: \mathrm{YAl}_{3}\left(\mathrm{BO}_{3}\right)_{4}$ laser," Opt. Commun. 195, 431-436 (2001).

19. A. Yoshida, A. Schmidt, V. Petrov, C. Fiebig, G. Erbert, J. Liu, H. Zhang, J. Wang, and U. Griebner, "Diodepumped mode-locked Yb:YCOB laser generating 35 fs pulses," Opt. Lett. 36, 4425-4427 (2011).

20. F. Druon, F. Balembois, P. Georges, A. Brun, A. Courjaud, C. Hönninger, F. Salin, A. Aron, F. Mougel, G. Aka, and D. Vivien, "Generation of 90 -fs pulses from a mode-locked diode-pumped $\mathrm{Yb}^{3+}: \mathrm{Ca}_{4} \mathrm{GdO}\left(\mathrm{BO}_{3}\right)_{3}$ laser," Opt. Lett. 25, 423-425 (2000).

21. Z.-Y. Gao, J.-F. Zhu, Z.-M. Wu, Z.-Y. Wei, H.-H. Yu, H.-J. Zhang, and J.-Y. Wang, "Tunable second harmonic generation from a Kerr-lens mode-locked $\mathrm{Yb}: \mathrm{YCa}_{4} \mathrm{O}(\mathrm{BO} 3)_{3}$ femtosecond laser," Chin. Phys. B 26, 044202/1-4 (2017).

22. M. Porębski, M. Kowalczyk, L. Gheorghe, M. Greculeasa, A. Broasca, F. Voicu, and J. Sotor, "Passively modelocked self-frequency doubling Yb:LGSB laser," in 2019 Conference on Lasers and Electro-Optics Europe and European Quantum Electronics Conference, OSA Technical Digest (Optical Society of America, 2019), paper ca_p_24.

23. M. J. Lederer, M. Hildebrandt, V. Z. Kolev, B. Luther-Davies, B. Taylor, J. Dawes, P. Dekker, J. Piper, H. H. Tan, and C. Jagadish, "Passive mode locking of a self-frequency-doubling $\mathrm{Yb}: \mathrm{YAl}_{3}\left(\mathrm{BO}_{3}\right)_{4}$ laser," Opt. Lett. 27, 436-438 (2002).

24. X. Zhang, Y. Zhou, J. Ren, D. Lu, H. Yu, Z. Wang, S. Guo, and X. Xu, "Growth, thermal and laser properties of a new self-frequency-doubling Yb:CNGS crystal," CrystEngComm 18, 5338-5343 (2016).

25. X. Zhang, P. Loiko, J. M. Serres, X. Mateos, J. Ren, Z. Wang, S. Guo, X. Xu, E. Vilejshikova, U. Griebner, V. Petrov, M. Aguiló, and F. Díaz, "Highly-efficient laser operation of a novel trigonal silicate crystal $\mathrm{Yb}^{3+}: \mathrm{Ca}_{3} \mathrm{NbGa}_{3} \mathrm{Si}_{2} \mathrm{O}_{14}, "$ Opt. Mater. Express 7, 3626-3633 (2017).

26. X. Zhang, P. Loiko, X. Mateos, J. M. Serres, J. Ren, J. Guo, R. Cheng, C. Gao, Q. Dong, V. Jambunathan, A. Lucianetti, T. Mocek, E. Vilejshikova, U. Griebner, V. Petrov, Z. Wang, S. Guo, X. Xu, M. Aguilo, and F. Diaz, "Crystal growth, low-temperature spectroscopy and multi-watt laser operation of $\mathrm{Yb}_{2} \mathrm{Ca}_{3} \mathrm{NbGa}_{3} \mathrm{Si}_{2} \mathrm{O}_{14}, " \mathrm{~J}$. Lumin. 197, 90-97 (2018).

27. P. Sévillano, P. Georges, F. Druon, D. Descamps, and E. Cormier, "32-fs Kerr-lens mode-locked Yb:CaGdAlO oscillator optically pumped by a bright fiber laser," Opt. Lett. 39, 6001-6004 (2014).

28. J. Ma, H. Huang, K. Ning, X. Xu, G. Xie, L. Qian, K. P. Loh, and D. Tang, "Generation of 30 fs pulses from a

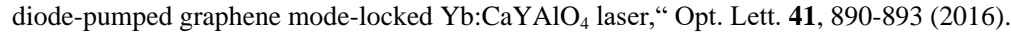

29. M. Kowalczyk, X. Zhang, X. Mateos, S. Guo, Z. Wang, X. Xu, P. Loiko, F. Rotermund, J. E. Bae, F. Rotermund, J. Sotor, U. Griebner, and V. Petrov, "Graphene and SESAM mode-locked Yb:CNGS lasers with self-frequency doubling properties," Opt. Express 27, 590-596 (2019).

30. M. Kowalczyk, A. Major, and J. Sotor, "High peak power ultrafast $\mathrm{Yb}: \mathrm{CaF}_{2}$ oscillator pumped by a singlemode fiber-coupled laser diode," Opt. Express 25, 26289-26295 (2017).

31. X. Zhang, X. Zhang, S. Guo, J. He, K. Han, F. Lou, B. Zhang, R. Wang, and X. Liu, "Growth and optical properties of a new CGG-type laser crystal Nd ${ }^{3+}$ :CNGS," Opt. Mater. Express 5, 977-985 (2015).

32. V. L. Kalashnikov, E. Podivilov, A. Chernykh, and A. Apolonski, "Chirped-pulse oscillators: Theory and experiment," Appl. Phys. B Lasers Opt. 83, 503-510 (2006).

33. M. Kowalczyk and J. Sotor, "Investigation on dispersion regimes in Yb:KGW solid-state laser," Laser Phys. Lett. 15, 065003/1-5 (2018). 
34. J. T. Manassah, "Effects of velocity dispersion on a generated second harmonic signal," Appl. Opt. 27, 43654367 (1988).

35. L. D. Noordam, H. J. Bakker, M. P. de Boer, and H. B. van Linden van den Heuvell, "Second-harmonic generation of femtosecond pulses: observation of phase-mismatch effects," Opt. Lett. 15, 1464-1466 (1990).

36. P. Trabs, F. Noack, A. S. Aleksandrovsky, A. I. Zaitsev, N. V. Radionov, and V. Petrov, "Spectral fringes in non-phase-matched SHG and refinement of dispersion relations in the VUV," Opt. Express 23, 10091-10096 (2015).

37. T. M. Kardaś, M. Nejbauer, P. Wnuk, B. Resan, C. Radzewicz, and P. Wasylczyk, "Full 3D modelling of pulse propagation enables efficient nonlinear frequency conversion with low energy laser pulses in a single-element tripler," Sci. Rep. 7, 42889/1-7 (2017).

38. F. Chen, F. Yu, S. Hou, Y. Liu, Y. Zhou, X. Shi, H. Wang, Z. Wang, and X. Zhao, "Crystal growth and characterization of CTGS and Nd:CTGS for self-frequency-doubling applications," CrystEngComm 16, 1028610291 (2014).

39. X. Zhang, Y. Zhou, A. Yasukevich, P. Loiko, X. Mateos, X. Xu, S. Guo, and Z. Wang, "Diode-pumped passively Q-switched self-frequency-doubled Nd:CNGS laser," Opt. Express 25, 19760-19766 (2017). 